\title{
DISCOVERY OF A NEW RADIO GALAXY WITHIN THE ERROR BOX OF THE UNIDENTIFIED GAMMA-RAY SOURCE 3EG J1735-1500
}

\author{
J. A. Combi, ${ }^{1}$ G. E. Romero, ${ }^{1}$ J. M. Paredes, ${ }^{2}$ D. F. Torres, ${ }^{3}$ and M. Ribó ${ }^{2}$ \\ Received 2002 November 20; accepted 2003 January 23
}

\begin{abstract}
We report the discovery of a new radio galaxy within the location error box of the $\gamma$-ray source 3EG J1735-1500. The galaxy is a double-sided jet source forming a large angle with the line of sight. Optical observations reveal a $V \sim 18$ mag galaxy at the position of the radio core. Although the association with the EGRET source is not confirmed at present because there is a competing, alternative $\gamma$-ray candidate within the location error contours, which we also study here, the case deserves further attention. The new radio galaxy can be used to test the recently proposed possibility of $\gamma$-ray-emitting radio galaxies beyond the already known case of Centaurus A.
\end{abstract}

Subject headings: galaxies: active — galaxies: jets — gamma rays: observations — radio continuum: galaxies

\section{INTRODUCTION}

The quest for the identification of the $\gamma$-ray sources detected by the EGRET instrument of the Compton Gamma-Ray Observatory is one of the most important challenges of high-energy astrophysics in recent years. Among the 271 sources included in the Third EGRET Catalog (3EG; Hartman et al. 1999) there are a few confirmed pulsars, 66 blazars, and a bunch of miscellaneous identifications including one radio galaxy: the nearby Centaurus A, a Fanaroff-Riley (FR) type I active galaxy (Sreekumar et al. 1999).

Most identified blazars are strong flat-spectrum radio sources (e.g., only four out of 46 high-probability blazar detections in the Mattox, Hartman, \& Reimer 2001 analysis have fluxes below $1 \mathrm{Jy}$ ) with jets pointing close to the line of sight. On the contrary, in the case of Centaurus A the viewing angle is rather large $\left(\sim 70^{\circ}\right.$; Bailey et al. 1986). Very recently, Mukherjee et al. (2002) have suggested that the radio galaxy NGC 6251 could also be associated with an EGRET source. If this is confirmed, it could have important consequences because the spatial density of FR I radio galaxies is far higher than that of blazars, and hence, despite that these objects are expected to be weaker $\gamma$-ray emitters, there could be many other unidentified sources associated with them.

In this paper we report the discovery of a new radio galaxy within the location error box of the $\gamma$-ray source 3EG J1735-1500. The object was found during a reanalysis of the main sources in the radio field around the position of the EGRET detection. This research is part of a systematic program to study potential low-energy counterparts of unidentified $\gamma$-ray sources. Previous results regarding compact radio sources were published by Torres et al. (2001b). Here we present the results of a more detailed study and new observations that led to the identification of the new radio

\footnotetext{
${ }^{1}$ Instituto Argentino de Radioastronomía, C.C.5, (1894) Villa Elisa, Buenos Aires, Argentina.

${ }^{2}$ Departament d'Astronomia i Meteorologia, Universitat de Barcelona, Avenida Diagonal 647, 08028 Barcelona, Spain.

${ }^{3}$ Lawrence Livermore National Laboratory, 7000 East Avenue, Livermore, CA 94550.
}

galaxy among the potential counterparts of 3EG J1735-1500. We also provide information on all compact radio sources within the EGRET location error box, including spectral index determinations when possible. We have also found that the source PMN J1738-1502 is probably a weak flat-spectrum quasar with mild radio flux, which enhances its probability of being the counterpart of 3EG J1735-1500 with respect to the a priori probability estimates by Mattox et al. (2001).

\section{RADIO DATA ANALYSIS AND RESULTS}

We have used the NRAO 4 VLA Sky Survey (NVSS; Condon et al. 1998) to study the small-scale radio emission within the inner $95 \%$ location probability contours of the $\gamma$-ray source 3EG J1735-1500. A total of 23 radio sources with flux density greater than $10 \mathrm{mJy}$ at $1.4 \mathrm{GHz}$ have been found, as can be seen in Figure 1, where we have numbered the sources with increasing Galactic longitude. The main characteristics of these sources are listed in Table 1. In particular, we provide, from left to right, the identification number corresponding to Figure 1, the Galactic coordinates, flux density at $1.4 \mathrm{GHz}$, spectral index (defined in such a way that $\left.S_{\nu} \propto \nu^{+\alpha}\right)$ when observations at other frequencies are available (365 MHz from Douglas et al. 1996 and $4.8 \mathrm{GHz}$ from Griffith \& Wright 1993), and the name in other catalogs, if any.

With the exceptions of sources 8 and 10 , all entries in Table 1 correspond to compact objects, at least at the present angular resolution $\left(43^{\prime \prime}\right)$. Source 8 , which appears as extended in our map, is actually a composite of three different (and probably compact) weak sources listed as 7, 8, and 9 in Torres et al. (2001b). Source 10, in contrast, is really extended. We shall name this source hereafter as J1737-15, according to its radio coordinates. Its morphology at 1.4 $\mathrm{GHz}$ (Fig. 2, upper panel) is typical of double-sided FR II radio galaxies. There is a nuclear component as well as two jets ending in large radio lobes. The angular dimensions of

\footnotetext{
${ }^{4}$ The National Radio Astronomy Observatory is a facility of the National Science Foundation operated under cooperative agreement by Associated Universities, Inc.
} 


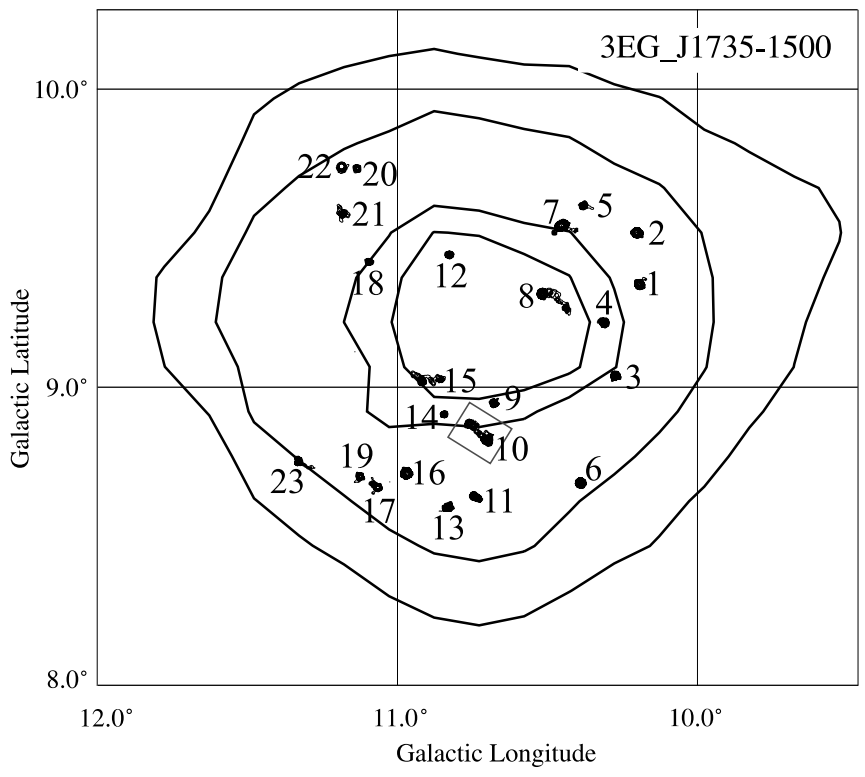

FIG. 1.-Gamma-ray probability contours $(50 \%, 68 \%, 95 \%$, and $99 \%$, from inside to outside) of the unidentified source 3EG 1735-1500, with the small-scale $1.4 \mathrm{GHz}$ emission obtained from the NVSS (where radio contours start at $1 \mathrm{mJy}$ beam $^{-1}$ ). The position of the radio galaxy is marked with a rectangle.

$\mathrm{J} 1737-15$ are $\sim 0.04 \times 0.11$, with an integrated flux of $55.6 \pm 1.5 \mathrm{mJy}$ at $1.4 \mathrm{GHz}$. The flux density of the northern component is $S_{\text {north }}=21.6 \pm 0.5 \mathrm{mJy}$, whereas the southern one is $S_{\text {south }}=30.7 \pm 1.3 \mathrm{mJy}$. The central component has a flux density of $S_{\text {core }}=3.80 \pm 0.05 \mathrm{mJy}$ and an estimated J2000.0 International Celestial Reference System (ICRS) position of $\alpha=17^{\mathrm{h}} 37^{\mathrm{m}} 12^{\mathrm{s}} .9 \pm 0.3, \delta=-15^{\circ} 11^{\prime} 02^{\prime \prime} \pm 15^{\prime \prime}$.

TABLE 1

Radio Sources Within the 95\% Gamma-Ray Contour

\begin{tabular}{|c|c|c|c|c|}
\hline Source & $\begin{array}{l}\text { Coordinates } \\
\qquad \begin{array}{c}(l, b) \\
(\mathrm{deg})\end{array}\end{array}$ & $\begin{array}{c}S_{1.4 \mathrm{GHz}} \\
(\mathrm{mJy})\end{array}$ & $\alpha$ & Other ID \\
\hline $1 \ldots \ldots \ldots \ldots$ & $(10.22,+9.34)$ & 71.00 & -1.34 & TXS $1731-153$ \\
\hline $2 \ldots \ldots \ldots \ldots$ & $(10.23,+9.51)$ & 128.96 & -1.2 & TXS $1730-152$ \\
\hline $3 \ldots \ldots \ldots .$. & $(10.29,+9.04)$ & 32.06 & $\ldots$ & $\ldots$ \\
\hline $4 \ldots \ldots \ldots . .$. & $(10.33,+9.21)$ & 31.68 & $\ldots$ & $\ldots$ \\
\hline $5 \ldots \ldots \ldots . .$. & $(10.39,+9.60)$ & 10.88 & $\ldots$ & $\ldots$ \\
\hline $6 \ldots \ldots \ldots .$. & $(10.41,+8.69)$ & 177.57 & -1.04 & TXS 1734-155 \\
\hline $7 \ldots \ldots \ldots . .$. & $(10.46,+9.53)$ & 133.77 & -0.54 & PMN J1734-1502 \\
\hline $8 \ldots \ldots \ldots \ldots$ & $(10.53,+9.31)$ & 169.72 & -0.89 & TXS 1732-150 \\
\hline 9............ & $(10.69,+8.95)$ & 24.74 & $\ldots$ & $\ldots$ \\
\hline $10 \ldots \ldots \ldots$ & $(10.74,+8.85)$ & 55.64 & $\lesssim-0.9$ & $\ldots$ \\
\hline $11 \ldots \ldots \ldots$ & $(10.76,+8.65)$ & 37.88 & $\ldots$ & $\ldots$ \\
\hline $12 \ldots \ldots \ldots$ & $(10.84,+9.43)$ & 17.07 & $\ldots$ & $\ldots$ \\
\hline $13 \ldots \ldots \ldots$ & $(10.84,+8.61)$ & 46.00 & $\ldots$ & $\ldots$ \\
\hline $14 \ldots \ldots \ldots$ & $(10.86,+8.91)$ & 11.33 & $\ldots$ & $\ldots$ \\
\hline $15 \ldots \ldots \ldots$ & $(10.94,+9.03)$ & 28.62 & $\ldots$ & $\ldots$ \\
\hline $16 \ldots \ldots \ldots$ & $(10.98,+8.72)$ & 330.40 & -0.17 & PMN J1738-1502 \\
\hline $17 \ldots \ldots \ldots$ & $(11.08,+8.68)$ & 19.15 & $\ldots$ & $\ldots$ \\
\hline $18 \ldots \ldots \ldots$ & $(11.11,+9.42)$ & 32.44 & $\ldots$ & $\ldots$ \\
\hline $19 \ldots \ldots \ldots$ & $(11.14,+8.71)$ & 10.73 & $\ldots$ & $\ldots$ \\
\hline $20 \ldots \ldots \ldots$ & $(11.15,+9.72)$ & 9.97 & $\ldots$ & $\ldots$ \\
\hline $21 \ldots \ldots \ldots$ & $(11.19,+9.58)$ & 9.63 & $\ldots$ & $\ldots$ \\
\hline $22 \ldots \ldots \ldots$ & $(11.19,+9.73)$ & 25.52 & $\ldots$ & $\ldots$ \\
\hline $23 \ldots \ldots \ldots$ & $(11.34,+8.76)$ & 38.43 & $\ldots$ & $\ldots$ \\
\hline
\end{tabular}

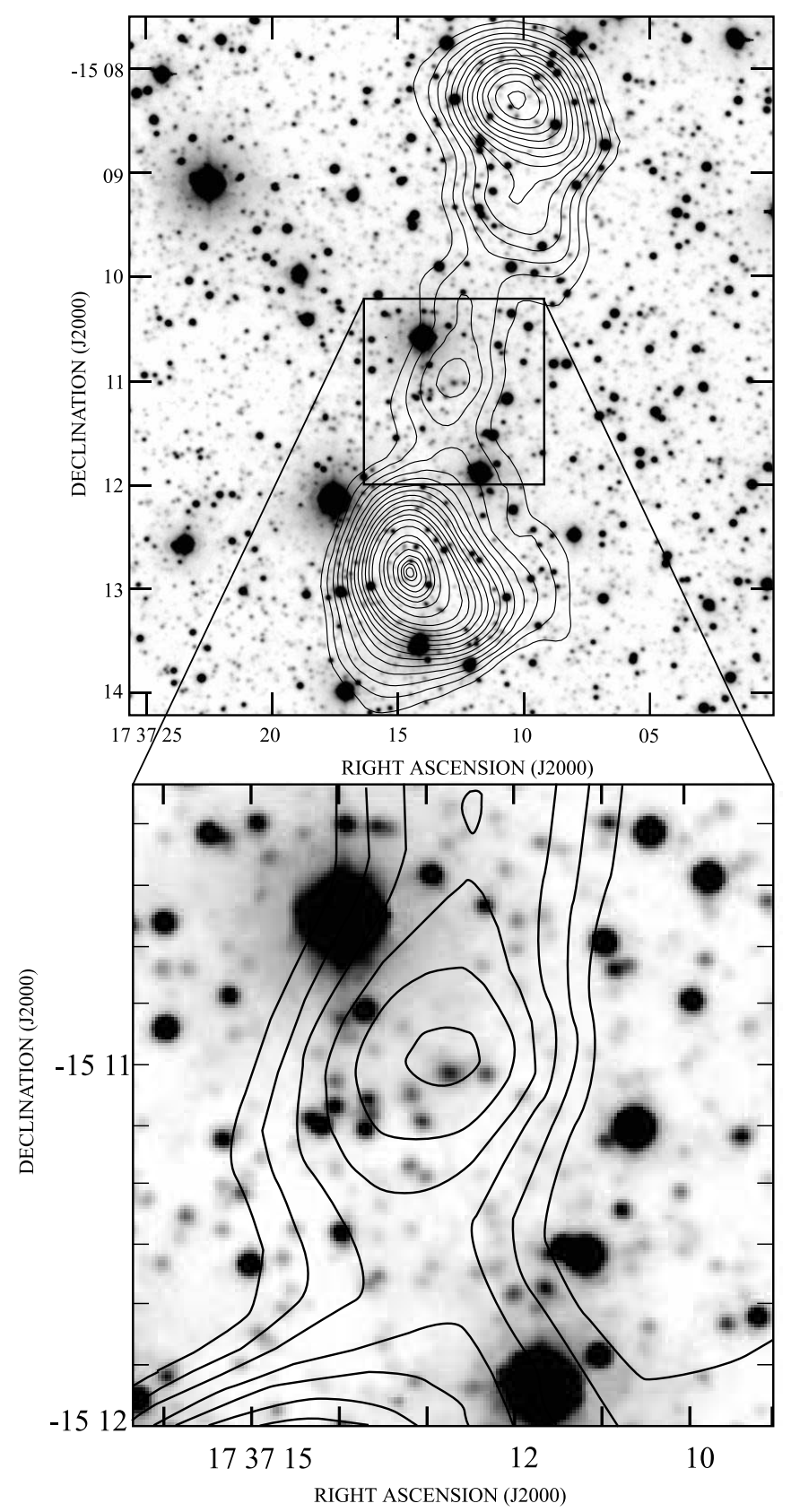

FIG. 2.-Upper panel: High-resolution radio image of the galaxy J1737-15 at $1.4 \mathrm{GHz}$ overlapped with the optical image obtained with the Calar Alto $2.2 \mathrm{~m}$ telescope using a Johnson $I$ filter. Radio contours are shown in steps of $0.6 \mathrm{mJy}$ beam $^{-1}$, starting from $1 \mathrm{mJy}^{\text {beam }}{ }^{-1}$. Lower panel: Enlargement of the central region of the radio galaxy showing the possible host galaxy. Radio contours are in steps of $0.3 \mathrm{mJy}^{\text {beam }}{ }^{-1}$, starting from $1.3 \mathrm{mJy}$ beam ${ }^{-1}$.

The source is clearly nonthermal since it is not detected at 4.8 GHz (Griffith \& Wright 1993). From the $4.8 \mathrm{GHz}$ survey sensitivity we can infer an average steep spectral index of $\alpha \lesssim-0.9$, but it is not possible to say, within the present resolution, what the index is at the core because of contamination from the radio lobes, which surely have steeper spectra.

The general morphology clearly supports the hypothesis of a radio galaxy with jets nearly perpendicular to the line of sight. Should the association between the radio galaxy and the EGRET source be confirmed, J1737-15 would be the 
second EGRET source (apart from Centaurus A) to be detected with a large inclination angle.

\section{OPTICAL OBSERVATIONS}

With the aim of finding the optical counterpart of the radio galaxy, we have made $V R I$ deep photometric observations of J1737-15 on 2002 June 9 at Calar Alto (Almería, Spain) with the $2.2 \mathrm{~m}$ telescope of the Centro Astronómico Hispano-Alemán (CAHA). Images were obtained using the Ritchey-Chrétien focus and the Calar Alto Faint Object Specrograph (CAFOS), with a scale factor of 0 ".53 pixel $^{-1}$ and an $8.8 \times 8.8$ field of view. A series of $600 \mathrm{~s}$ images of our target, as well as $200 \mathrm{~s}$ images of standard stars from the SA104 field of Landolt (1992), were obtained through the $V$, $R$, and $I$ Johnson filters. The observations were reduced using standard procedures (bias and dark subtraction, and flat-field correction) within the IRAF software package. We performed a detailed astrometric reduction of the images using 20 field stars present in the USNO-A2.0 catalog (Monet et al. 1999), with an estimated astrometric accuracy of 0 !" 3 .

We show in Figure 2 the image obtained through the I Johnson filter, together with the contours of the radio source from the NVSS. It is clear at first sight that there is a slightly elongated (east-west) optical object close to the center of the radio source. The fitted J2000.0 ICRS position of this object is $\alpha=17^{\mathrm{h}} 37^{\mathrm{m}} 12^{\mathrm{s}} .744 \pm 0.021$, $\delta=-15^{\circ} 11^{\prime} 01^{\prime \prime} .14 \pm 0$ "' 30 , well within the previously obtained error box in position for the core of the radio source. We also obtained absolute photometry of this object, which is believed to be accurate only to $\pm 0.2 \mathrm{mag}$, because the crowded field around our target prevented a good estimate of the background. The obtained magnitudes are $V=$ $18.3 \pm 0.2, R=16.9 \pm 0.2$, and $I=15.1 \pm 0.2$. Using the Galactic extinction estimates from Schlegel, Finkbeiner, \& Davis (1998), implemented within the NASA/IPAC Extragalactic Database (NED), we obtain a color excess of $E(B-V)=0.495$ in the direction of $\mathrm{J} 1737-15$. However, as pointed out by Arce \& Goodman (1999), the values provided by the Schlegel model could be overestimated by a factor of 1.3-1.5 in regions of smooth extinction with $E(B-V)>0.15 \mathrm{mag}$, as it happens in our case. As a reasonable value, we have considered that the NED value is overestimated by a factor of $1.3 \pm 0.2$ and used an extinction of $E(B-V)=0.38 \pm 0.05$. This provides the following extinctions: $A_{V}=3.315, E(B-V)=1.3 \pm 0.2 ; A_{R}=2.673$, $E(B-V)=1.0 \pm 0.1 ;$ and $A_{I}=1.940, E(B-V)=0.7 \pm 0.1$ (Schlegel et al. 1998). Using these values we obtain the following dereddened magnitudes: $V=17.0 \pm 0.3, R=15.9$ \pm 0.2 , and $I=14.4 \pm 0.2$. These values imply color indices of $V-R=1.1 \pm 0.3$ and $R-I=1.5 \pm 0.3$ (where the errors have been computed directly from the uncertainties in measured magnitudes and assumed $E(B-V)$.

If we suppose that this optical object is a star, the ranges of obtained dereddened colors only allow possibilities such as M3 III or M0 I, according to Ducati et al. (2001). However, comparison of the obtained apparent dereddened visual magnitude with the expected absolute visual magnitude (Wainscoat et al. 1992) implies distances of 33 and $465 \mathrm{kpc}$, respectively. The second value is unrealistic, while the first one implies a Galactic height $\left(b=9^{\circ} .2\right)$ of $\sim 5 \mathrm{kpc}$, in clear disagreement with what is typically found for M3 III stars. Therefore, the dereddened colors are clearly incompatible with the object being a single Galactic star located by chance in the same direction as the radio galaxy core.

On the other hand, since the source is elongated, a twodimensional Gaussian fit can provide information about the ellipticity and position angle. We have performed several Gaussian fits using different fitting-box sizes. In all cases the ellipticity of the source seems to be around 0.14 , while the position angle changes from $85^{\circ}$ (counterclockwise from north) to $75^{\circ}$, when reducing the size of the fitting box. In any case, the object is extended practically in the east-west direction, i.e., perpendicular to the radio jets. All these facts suggest that our identified optical counterpart is a galaxy, at the center of which the jets visible at radio wavelengths are produced.

In addition to the optical counterpart we have looked for infrared and X-ray sources at the position of J1737-15. Filtered IRAS images (Wheelock et al. 1991) at 12 and $100 \mu \mathrm{m}$ of the region show no particular infrared enhancement at the central position of the radio galaxy. The ROSAT AllSky Survey $(0.1-2.4 \mathrm{keV})$ shows three X-ray sources inside of the $\gamma$-ray $95 \%$ contour, but none of them is close to J1737-15.

\section{DISCUSSION}

Depending on the jet and ambient medium parameters, most double-sided radio sources have sizes below $\sim 300 \mathrm{kpc}$ (Begelman, Blandford, \& Rees 1984). In the case of the radio galaxy reported here, and using standard FriedmannRobertson-Walker formulae, this size translates into a possible distance smaller than $350 \mathrm{Mpc}$. If 3EG J1735-1500 is indeed the result of $\gamma$-ray emission in $\mathrm{J} 1737-15$, the intrinsic luminosity at $E>100 \mathrm{MeV}$, at the distance quoted, should be smaller than $2 \times 10^{44} \mathrm{ergs} \mathrm{s}^{-1}$, although mild beaming could reduce this figure. A correct determination of the distance requires a knowledge of the redshift that is still unknown. The information we provide here for the optical counterpart will be useful in guiding future spectroscopic observations. As in the case of Centaurus A and 3EG $\mathrm{J} 1621+8203$, the luminosity needed to generate the observed $\gamma$-ray source is several orders of magnitude less than those required for typical EGRET blazars $\left(10^{45}-10^{48}\right.$ ergs $\mathrm{s}^{-1}$ ).

The $\gamma$-ray variability history of 3EG J1735-1500 is shown in Figure 3. The source was detected in only three EGRET viewing periods (VPs) - in the remaining VPs only upper limits to the flux were established - and appears to be variable. Torres et al.'s (2001b) index $I$ gives $I=8.86$, which identifies this source as one of the most variable $\gamma$-ray sources in the EGRET catalog. Tompkins' (1999) index $\tau$ is $\tau=1.09_{0.00}^{10.1}$. Although inconclusive because of its lower limit, its central value and upper limit also identify this source as one of the most variable in the catalog. (For a detailed discussion on variability indices see Torres, Pessah, $\&$ Romero 2001a). The likely variability status of this source might imply that the $\gamma$-ray emission is produced in the central region and not in the extended lobes, if related to the discovered radio galaxy. The photon spectral index seems to be unusually steep with a value $\Gamma=3.24 \pm 0.47$, steeper than the average of the $\gamma$-ray blazars spectra, a property also shared by Centaurus A and 3EG J1621+8203.

Since 3EG J1735-1500 is at a low Galactic latitude, it could be possible that some Galactic object not visible at radio wavelengths is responsible for the high-energy 


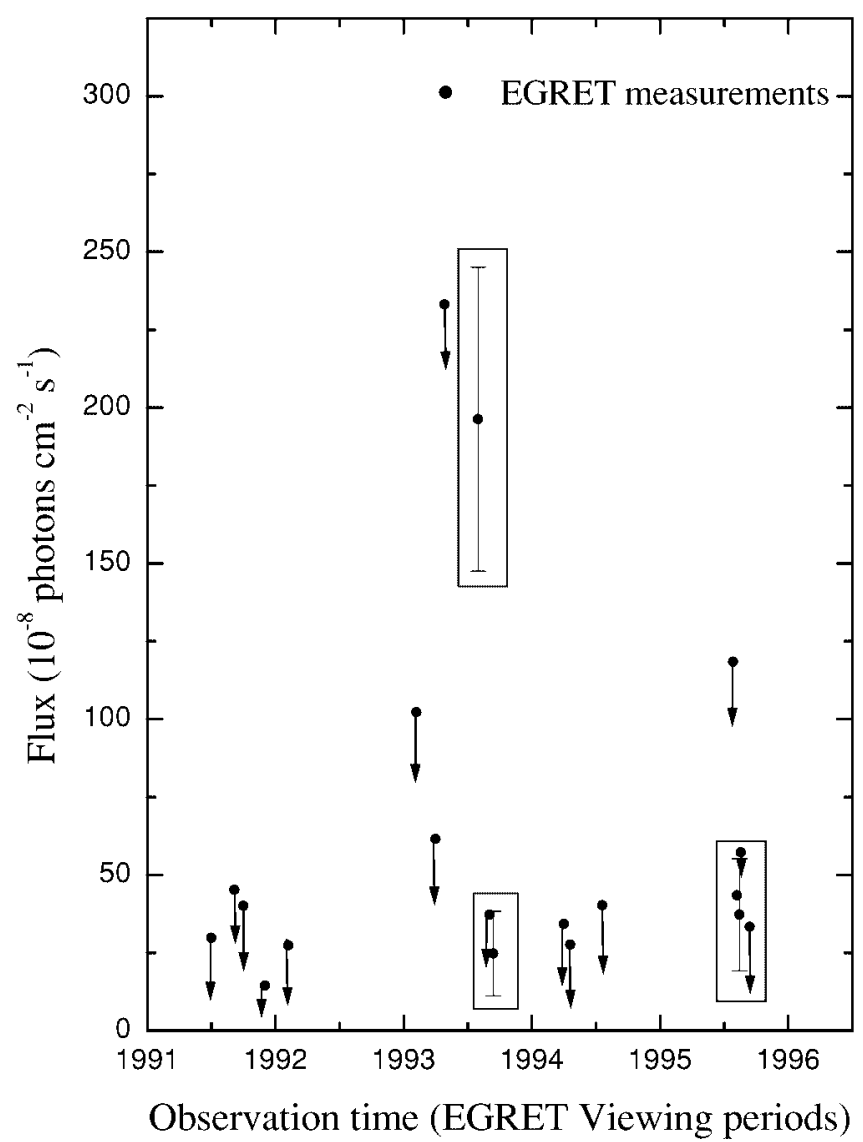

FIG. 3.-Gamma-ray history of 3EG J1735-1500. Boxes indicate source detections as opposed to upper limits.

emission. However, the source is not coincident with earlytype stars, OB associations, or galactic X-ray binaries (Romero, Benaglia, \& Torres 1999).

The only other known potential counterpart in addition to the galaxy discussed here is the source PMN $\mathrm{J} 1738-1502$. This is a compact radio source with a total flux at $1.4 \mathrm{MHz}$ of $\sim 0.3 \mathrm{Jy}$ (source 16 in Table 1 ). We have calculated a flat spectral index for this object $\alpha=-0.17$, which makes of it a clear blazar candidate. Mattox et al. (2001) give an a priori probability of only $0.07 \%$ for a physical association. The a posteriori probability (see Mattox et al. 2001 for details) is slightly higher but still very small: $0.35 \%$. The radio source is weaker than most high-probability $\gamma$-ray blazars, but similar to most plausible (i.e., with lower a priori probability) identifications. Consequently, this source should be considered a serious alternative counterpart of the EGRET source, despite the low probabilities given by Mattox et al. (2001). The object is similar to the plausible identification proposed recently by Wallace et al. (2002) in the case of 3EG J2006-2321, although we notice that in the case of 3EG J1735-1500 the high-energy photon index is far softer $(\Gamma=3.24 \pm 0.47$ vs. $\Gamma=2.47 \pm 0.44)$. Since $\gamma$-ray blazars tend to be highly polarized and variable, we are planning optical polarization observations of this source in order to confirm its blazar nature.

One of the main problems in explaining the synchrotron emission in radio galaxies is that this radiation is distributed rather uniformly along distances of $\sim 100 \mathrm{kpc}$. To explain how the continuous acceleration of electrons takes place in these huge sources remains as one of the most difficult topics in jet theory. Recently, it has been suggested by Neronov et al. (2002) that in some cases the relativistic leptonic population can be locally created by very high energy $\gamma$-rays produced at the central source and injected into the jet, where they produce pairs through interactions with the $2.7 \mathrm{~K}$ cosmic background radiation. In this scenario lower energy $(\mathrm{GeV})$ photons escape from the radio galaxy and could even traverse the diffuse infrared background without further interaction before reaching the Earth. The $\gamma$-rays could be produced in the central engine by a photon-pion process involving disk photons (Neronov et al. 2002). Future multifrequency observations of $\mathrm{J} 1737-15$ could help to test the proposal of an association with 3EG J1735-1500. If the high-energy emission is confirmed, this radio galaxy could become an important natural laboratory to test the theories of nonthermal emission in extragalactic jet sources.

\section{CONCLUSIONS}

We have discovered a new radio galaxy inside the error box of the $\gamma$-ray source 3EG J1735-1500. It is a doublesided FR II type of object. Its linear angular size implies that it is relatively nearby. We have also identified the host optical galaxy, which appears elongated in a direction perpendicular to the radio structure. Future spectroscopic observations will help to fix the distance to this object. If it turns out to be closer than $100 \mathrm{Mpc}$, the radio galaxy could be a potential acceleration site of ultra-high-energy cosmic rays, avoiding the so-called Greisen-Zatsepin-Kuz'min (GZK) cutoff (see Rachen \& Biermann 1993).

We have also studied the other important likely potential counterpart of $3 E G \mathrm{~J} 1735-1500$. We found that it is a flat-spectrum compact radio source, possibly a blazar, and hence perhaps a $\gamma$-ray-emitting object. The remaining sources within the location confidence contours of the EGRET detection seem to be uninteresting from the point of view of the high-energy emission. Forthcoming observational studies of these two objects will shed light on the real nature of 3EG J1735-1500.

This work was partially supported by the Argentinian agencies Consejo de Investigaciones Científicas y Técnicas (CONICET PIP 0430/98) and Agencia Nacional de Promoción Científica y Técnica (ANPCT PICT 03-04881), as well as by the DGI of the Ministerio de Ciencia y Tecnología (Spain) under grant AYA 2001-3092 and the European Regional Development Fund (ERDF/FEDER). Additional support was provided by Fundación Antorchas. The work of D. F. T. was performed under the auspices of the US Department of Energy, National Nuclear Security Administration, by the University of California Lawrence Livermore National Laboratory under contract W-7405Eng-48. M. R. is supported by a fellowship from CIRIT (Generalitat de Catalunya, ref. 1999 FI 00199). We thank the German-Spanish Astronomical Centre, Calar Alto, operated by the Max-Planck-Institut für Astronomie (Heidelberg) jointly with the Spanish National Commission for Astronomy, for carrying out the observations under the Director's Discretionary Time Service. 
Arce, H. G., \& Goodman, A. A. 1999, ApJ, 512, L135

\section{REFERENCES}

Bailey, J., et al. 1986, Nature, 322, 150

Begelman, M. C., Blandford, R. D., \& Rees, M. J. 1984, Rev. Mod. Phys., 56,255

Condon, J. J., et al. 1998, AJ, 115, 1693

Douglas, J. N., et al. 1996, AJ, 111, 1945

Ducati, J. R., Bevilacqua, C. M., Rembold, S. B., \& Ribeiro, D. 2001, ApJ, 558,309

Griffith, M. R., \& Wright, A. E. 1993, AJ, 105, 1666

Hartman, R. C., et al. 1999, ApJS, 123, 79

Landolt, A. U. 1992, AJ, 104, 340

Mattox, J. R., Hartman, R. C., \& Reimer, O. 2001, ApJS, 135, 155

Monet, D. G., et al. 1999, USNO-A2.0 CD-ROM (Washington: USNO)

Mukherjee, R., Halpern, J., Mirabal, N., \& Gotthele, E. V. 2002, ApJ, 574, 693

Neronov, A., Semikoz, D., Aharonian, F., \& Kalashev, O. 2002, Phys. Rev. Lett., 89,1101

Rachen, J. P., \& Biermann, P. L. 1993, A\&A, 272, 161

Romero, G. E., Benaglia, P., \& Torres, D. F. 1999, A\&A, 348, 868

Schlegel, D. J., Finkbeiner, D. P., \& Davis, M. 1998, ApJ, 500, 525

Sreekumar, P., et al. 1999, Astropart. Phys., 11, 221

Tompkins W. 1999, Ph.D. thesis, Stanford Univ.

Torres, D. F., Pessah, M. E., \& Romero, G. E. 2001a, Astron. Nachr., 322, 223

Torres, D. F., et al. 2001b, A\&A, 370, 468

Wainscoat, R. J., Cohen, M., Volk, K., Walker, H. J., \& Schwartz, D. E. 1992, ApJS, 83, 111

Wallace, P. M., Halpern, J. P., Magalhães, A. M., \& Thompson, D. J. 2002, ApJ, 569, 36

Wheelock, S., et al. 1991, BAAS, 23, 908 